\title{
MULTIPLICATORS AND METABELIAN GROUPS
}

\author{
GILBERT BAUMSLAG*
}

(Received 20 May 1975)

\begin{abstract}
We prove here that the multiplicator of certain simply described metabelian groups is not finitely generated.
\end{abstract}

\section{Introduction}

This note grew out of an attempt to discern which finitely generated metabelian groups are finitely presented. The complexity of finitely generated metabelian groups, however, suggests that this is no easy task (see Baumslag (1974) for an overall discussion of this topic). In particular the multiplicator (i.e. the second homology group with integral coefficients) is not sufficiently discriminating to distinguish which of the finitely generated metabelian groups are finitely presented since there exist finitely generated metabelian groups with trivial multiplicator which are not finitely related (Baumslag (1971); cf. also Baumslag \& Strebel (in press)). Our main objective here is to show that the multiplicators of some simply described finitely generated metabelian groups are not finitely generated, thereby demonstrating that these groups are not finitely presented.

In order to explain our first two theorems we shall write

$$
G=\left\langle\left\langle a_{1}, \cdots, a_{p} ; r_{1}=1, \cdots, r_{q}=1\right\rangle\right\rangle
$$

to express the fact that the group $G$ is generated by its elements $a_{1}, \cdots, a_{p}$ and defined in terms of these elements by all relations of the form $[[w, x],[y, z]]=1$ together with the exhibited relations $r_{1}=1, \cdots, r_{q}=1$ (here $[u, v]=u^{-1} v^{-1} u v$ and, for later use, $u^{v}=v^{-1} u v$ ). We shall term $G$ a $q$-relator metabelian group in order to underline the fact that it can be presented in this way.

We shall prove, in 2 , the

*Support from the National Science Foundation, the hospitality of Warwick University and the Institute of Advance Study of the Australian National University is gratefully acknowledged. 
THEOREM 1. Let

$$
G=\left\langle\left\langle a_{1}, \cdots, a_{p} ; r_{1}=1, \cdots, r_{q}=1\right\rangle\right\rangle .
$$

If $p-q>1$ then the multiplicator $m(G)$ of $G$ is not finitely generated.

It follows immediately from Theorem 1 that if $G=$ $\left\langle\left\langle a_{1}, \cdots, a_{p} ; r_{1}=1, \cdots, r_{q}=1\right\rangle\right\rangle$ and $\left.p-q\right\rangle 1$, then $G$ is not finitely presented. Hence, in particular, it follows also that free metabelian groups are finitely presented only if cyclic (Šmel'kin (1965)).

The proof of Theorem 1 can be readily adapted to give us

THEOREM 2. Let $G=\left\langle\left\langle a_{1}, \cdots, a_{p} ; r=1\right\rangle\right\rangle$ be a 1-relator metabelian group that is not cyclic. Then, if either $p>2$ or $r$ is a proper power, $m(G)$ is not finitely generated (and hence, again, $G$ is not finitely presented).

Notice that the case $p>2$ is covered by Theorem 1 .

It is perhaps worth emphasizing that it follows from Theorems 1 and 2 that if $H$ is a group presented on $p$ generators and $q$ defining relations, then $H / H^{\prime \prime}$ is not finitely related if either $p-q>1$ or $p>1$ and $r$ is a proper power (here $H^{\prime \prime}$ denotes the second derived group of $H$ ). In particular if $H$ is a group with a single defining relation, then $H / H^{\prime \prime}$ cannot be finitely presented unless it is generated by two elements, in which case almost anything can occur (cf. Baumslag (1974)).

Let $G_{n}$ denote the $n$th term of the lower central series of the group $G$ and let $G_{\omega}=\bigcap_{n=1}^{\infty} G_{n}$. Further, let $\hat{G}$ denote the pronilpotent completion of the group $G$, i.e. the limit of the inverse system consisting of the groups $G / G_{n}$ and the natural homomorphisms from $G / G_{n}$ onto $G / G_{m}(n>m)$. Then we shall prove, in 3 , the

THEOREM 3. Let $F$ be a finitely generated free metabelian group, $G$ a finitely generated metabelian group. If $G / G_{\omega}$ is isomorphic to a non-abelian subgroup of $\hat{F}$ then $m(G)$ is not finitely generated.

It follows immediately from Theorem 3 that

Corollary 1. Let $G$ be a finitely generated metabelian group and suppose that $G / G_{n} \cong F / F_{n}(n=1,2, \cdots)$, where $F$ is a non-abelian free metabelian group. Then $m(G)$ is not finitely generated (and so $G$ is not finitely presented).

A residually nilpotent group satisfying the hypothesis of Corollary 1 is termed parafree metabelian (Baumslag (1969)). Thus it follows that we have proved the 
CoROllary 2. A non-cyclic parafree metabelian group is not finitely presented.

Remeslennikov (1969) has shown that a finitely generated metabelian group contains a subgroup of finite index which is residually nilpotent. This suggests that if $G$ is a finitely generated but not finitely related metabelian group, then $G$ contains a subgroup of finite index whose multiplicator is not finitely generated. This is false, however, and we shall give an appropriate example in 4.

\section{The proofs of Theorems 1 and 2}

The proof of Theorem 1 (and also that of Theorem 2) depends on two simple lemmas; the first of these is contained in Baumslag (1972).

Lemma 1. Let $G$ be a finitely generated metabelian group. Then $m(G)$ is finitely generated if and only if $m(H)$ is finitely generated for every quotient group $H$ of $G$.

The second lemma is the almost obvious

Lemma 2. Let $R$ be an integral domain (commutative, with 1) and let $M$ be a free $R$-module of finite rank $n$. If $N$ is an $(n-1)$-generator submodule of $M$, then $M / N$ is not a torsion module.

Proof. Suppose that $M / N$ is a torsion module. Let $F$ be the quotient field of $R$. Then $M \otimes F$ is an $F$-vector space of dimension $n$. Since $M / N$ is a torsion-module, $N \otimes F=M \otimes F$. But $N \otimes F$ can be generated as an $F$-vector space by $n-1$ elements, which implies that $M \otimes F$ is of dimension at most $n-1$. This contradiction completes the proof of Lemma 2 .

We can now prove Theorem 1 . Thus suppose that

$$
G=\left\langle\left\langle a_{1}, \cdots, a_{p} ; r_{1}=1, \cdots, r_{q}=1\right\rangle\right\rangle
$$

where $p-q>1$. By the basis theorem for free abelian groups we can present $G$ in a more suitable form

$$
G=\left\langle\left\langle t, a, b, \cdots, c ; b^{\beta} v=1, \cdots, c^{\gamma} w=1\right\rangle\right\rangle,
$$

where in the new defining relations the words $v, \cdots, w$ lie in the commutator subgroup and $\beta, \cdots, \gamma$ are integers (possibly 0 ). Let $A=g p_{G}(a, b, \cdots, c$ ) be the normal closure in $G$ of the elements $a, b, \cdots, c$ and put

$$
P=A / A_{2} A^{\prime}
$$

where $L$ is an arbitrarily chosen prime and $A^{\prime}$ is the subgroup of $A$ generated by its $/$ th powers. Let $R$ be the group algebra of the infinite cyclic group $\langle t\rangle$ over 
the field of $l$ elements. Then $P$ may be thought of as the quotient of a free $R$-module $M$ of rank $p-1$ by a submodule $N$ generated by $q$ elements. Since $q \leqq(p-1)-1$, Lemma 2 applies here and hence $P$ is not a torsion module. Now $P$ is a finitely generated $R$-module and $R$ is a principal ideal domain. Therefore $P$ is a direct sum of a finite number of cyclic submodules. Since $P$ is not a torsion module at least one of these submodules is torsion-free.

On putting these facts together it follows readily that $G / A_{2} A^{\prime}$ has a factor group which is isomorphic to $W=C_{1}$ wr $c$, the wreath product of cyclic group of order $l$ by an infinite cyclic group. But $m(W)$ is not finitely generated (Baumslag (1972)). So by Lemma $1 m(G)$ is not finitely generated either. This completes the proof of Theorem 1.

We come now to the proof of Theorem 2. Thus suppose that $G=$ $\left\langle\left\langle a_{1}, \cdots, a_{p} ; r=1\right\rangle\right\rangle$. If $p>2$, then Theorem 1 applies and $m(G)$ is not finitely generated. If $p=2$, then by hypothesis, $r$ is a proper power, say $r=r_{1}^{s}$ with $s>1$. We choose now a second presentation for $G$ :

$$
G=\left\langle\left\langle t, a,\left(a^{\alpha} v\right)^{s}=1\right\rangle\right\rangle,
$$

where $v$ lies in the derived group and $\alpha$ is an integer. As in the proof of Theorem 1 let $A=g p_{G}(a)$ and put $P=A / A_{2} A^{\prime}$ where now $l$ is chosen to be a prime dividing $s$. Then it is not hard to see that $P$ is actually a free $R$-module, with $R$ as before. So $G / A_{2} A^{\prime} \cong C_{l}$ wr $C$, which means that $m(G)$ is not finitely generated as required.

\section{The proof of Theorem 3}

The proof of Theorem 3 is motivated by the following well-known result.

Lemma 3. Let $G$ be a finitely generated group. Then $m(G)$ is finitely generated if and only if the central subgroup $C$ in every finitely generated central extension $E$ of the form

$$
1 \rightarrow C \rightarrow E \rightarrow G \rightarrow 1
$$

is finitely generated.

Next we need to recall a result from Baumslag (1969). To this end let $F$ be a free metabelian group of finite rank $n$, freely generated by $a_{1}, \cdots, a_{n}$ and let $\hat{F}$ be its pronilpotent completion. Then $\hat{F}$ is a parafree metabelian group with $\hat{F} /(\hat{F})_{2}$ free abelian of rank $n$ (Bousfield \& Kan (1971)). $F$ is naturally embedded in $\hat{F}$ and so we may think of $a_{1}, \cdots, a_{n}$ as elements of $\hat{F}$. Now let $R$ be the ring of power series with integral coefficients in the commuting variables $x_{1}, \cdots, x_{n}$ and let $\Lambda$ be the polynomial ring over $R$ in the commuting variables $y_{1}, \cdots, y_{n}$. Then it follows from the results of Baumslag (1969) that 
LEMMA 4. The mappings

$$
\theta: a_{i} \mapsto\left(\begin{array}{cc}
x_{i}+1 & 0 \\
y_{i} & 1
\end{array}\right) \quad(i=1, \cdots, n)
$$

and

$$
\phi: a_{i} \mapsto\left(\begin{array}{cc}
x_{i}+1 & y_{i} \\
0 & 1
\end{array}\right) \quad(i=1, \cdots, n)
$$

can be continued to monomorphisms, denoted respectively again by $\theta$ and $\phi$, of $\hat{F}$ into the $2 \times 2$ general linear group $G L(2, \Lambda)$ over the ring $\Lambda$.

Suppose now that $F \in \hat{F}$. Then, putting

$$
f \theta=\left(\begin{array}{cc}
f^{\prime} & 0 \\
\prime \prime & 1
\end{array}\right) \text { and } f \phi=\left(\begin{array}{cc}
f^{\prime} & f^{\prime \prime} \\
0 & 1
\end{array}\right)
$$

where of course $f^{\prime}$, " $f$ and $f$ " are elements of $\Lambda$ depending on $f$, we have

Corollary 4.1. If $f \in \hat{F}$ and $f^{\prime}=1$, then " $f=0$ if and only if $f "=0$.

Corollary 4.1 is an obvious consequence of the fact that $\theta$ and $\phi$ are monomorphisms. For if $f^{\prime}=1$ and " $f=0$, then $f \theta=1$. So $f \phi=1$ which means that $f^{\prime \prime}=0$.

Consider now the subgroup $T$ of the $3 \times 3$ general linear group $G L(3, \Lambda)$ over $\Lambda$ consisting of the triangular matrices (first introduced by Gupta (1969)) of the form

$$
\left(\begin{array}{lll}
1 & 0 & 0 \\
\alpha & \beta & 0 \\
\gamma & \delta & 1
\end{array}\right) \quad(\beta \text { a unit in } R)
$$

It is easy to check that the center $Z$ of $T$ consists of matrices of the form

$$
\left(\begin{array}{lll}
1 & 0 & 0 \\
0 & 1 & 0 \\
\gamma & 0 & 1
\end{array}\right)
$$

It follows directly from Lemma 3 and the way in which matrices in $T$ multiply that

Corollary 4.2 If $\mu$ is the mapping of $\hat{F}$ into $T$ defined by

$$
\mu: f \mapsto\left(\begin{array}{ccc}
1 & 0 & 0 \\
" f & f^{\prime} & 0 \\
0 & f^{\prime \prime} & 1
\end{array}\right) \quad(f \in \hat{F}),
$$

then the induced mapping 


$$
f \mapsto f \mu Z(f \in \hat{F})
$$

is a monomorphism of $\hat{F}$ into $T / Z$.

Next we prove the simple

LeMMA 5. Let

$$
A=\left(\begin{array}{lll}
1 & 0 & 0 \\
\alpha & 1 & 0 \\
\gamma & \beta & 1
\end{array}\right) \text { and } B=\left(\begin{array}{lll}
1 & 0 & 0 \\
\delta & \rho & 0 \\
\gamma & \varepsilon & 1
\end{array}\right),
$$

where $\rho$ is an invertible element of $R$ and $\alpha, \beta, \gamma, \delta, \varepsilon, \gamma$ belong to $\Lambda$. If $\rho$ is not a rational integer and $\alpha, \beta$ are non-zero, then $g p(A, B)$ has an infinitely generated center.

Proof. We simply compute the commutators

$$
C_{i}=\left[A, B^{-i} A B^{-i}\right] \quad(i=1,2, \cdots) .
$$

It turns out that

$$
C_{i}=\left(\begin{array}{ccc}
1 & 0 & 0 \\
0 & 1 & 0 \\
\gamma_{i} & 0 & 1
\end{array}\right)
$$

where

$$
\gamma_{i}=\alpha \beta\left(\rho^{-i}-\rho^{i}\right) \quad(i=1,2, \cdots) .
$$

Since the subring of $R$ generated by $\rho$ and $\rho^{-1}$ is simply the group ring of the infinite cyclic group over the ring of rational integers, it follows that the $\gamma_{i}$ are additively linearly independent. Therefore the elements $C_{1}, C_{2}, \cdots$ freely generate a free abelian group of infinite rank. This completes the proof of the lemma.

We are now in a position to prove Theorem 3. Thus suppose that $G$ is a finitely generated metabelian group such that $G / G_{\omega}$ is isomorphic to a nonabelian subgroup of $\hat{F}$. It follows from Lemma 1 that it is enough to prove that $\left(G / G_{\omega}\right)$ is not finitely generated. Consequently we may assume that $G$ is itself a subgroup of $\hat{F}$.

We select a finite set $g_{1}, \cdots, g_{m}$ of generators of $G$ and, employing the notation introduced above, then define $E$ to be the matrix group generated by the matrices

$$
\left(\begin{array}{ccc}
1 & 0 & 0 \\
" g_{1} & g_{1}^{\prime} & 0 \\
0 & g_{1}^{\prime \prime} & 1
\end{array}\right), \cdots,\left(\begin{array}{ccc}
1 & 0 & 0 \\
" g_{m} & g_{m}^{\prime} & 0 \\
0 & g_{m}^{\prime \prime} & 1
\end{array}\right) .
$$


It follows from Corollary 4.2 that $E$ is a finitely generated central extension of $Z \cap E$ by $G$ :

$$
1 \rightarrow Z \cap E \rightarrow E \rightarrow G \rightarrow 1 .
$$

Thus in order to prove that $m(G)$ is not finitely generated it suffices, by Lemma 3 , to show that $Z \cap E$ is not finitely generated.

To this end we choose matrices $A, B \in E$ such that $A \notin Z, A \in E_{2}$, $B \notin E_{2} Z$. It follows on appealing to Corollary 4.1 that

$$
A=\left(\begin{array}{ccc}
1 & 0 & 0 \\
\alpha & 1 & 0 \\
\gamma & \beta & 1
\end{array}\right) \text { and } B=\left(\begin{array}{ccc}
1 & 0 & 0 \\
\delta & \rho & 0 \\
\gamma & \varepsilon & 1
\end{array}\right)
$$

where $\alpha, \beta$ are non-zero and $\rho$ is not a rational integer. Consequently $H=g p(A, B)$ does not have a finitely generated center, by Lemma 5 . But the center of $H$ is contained in $Z$. Therefore $Z \cap E$ is not finitely generated, as required.

\section{An example}

Remeslennikov (1969) has proved that if $G$ is a finitely generated metabelian group, then $G$ contains a subgroup of finite index which is residually nilpotent. This raises the possiblility that if $G$ is a finitely generated metabelian group which is not finitely related, then $G$ contains a subgroup of finite index whose multiplicator is not finitely generated. However this is not the case. Indeed we have the following

EXAmple. Let $G=\left\langle\left\langle a, b, s ; a^{s}=a^{4}, b^{s-1}=b^{8}\right\rangle\right\rangle$. Then $G$ is not finitely pre sented but every subgroup of finite index in $G$ has finitely generated multiplicator.

Actually examples of this type abound - many of the groups considered by Baumslag and Strebel (in press) have the same property as $G$.

It is a little troublesome to prove that $G$ has the properties stated. We shall only indicate the proof. First we note that if a group has a finitely generated multiplicator, so does every finite extension (Baumslag and Strebel (in press)) incidentally the converse is false. It suffices therefore to verify that normal subgroups of finite index in $G$ have finitely generated multiplicator. Indeed it follows from the same remark that it is enough to prove that the normal subgroups of the type

$$
g p\left(a^{\prime}, b^{t}, s^{m}\right)
$$

have finitely generated multiplicator. But this follows without difficulty by an analogous argument to that used in Baumslag (1971). 


\section{References}

Gilbert Baumslag (1969), 'Groups with the same lower central sequence as a relatively free group. 1 II. Properties', Trans. Amer. Math. Soc. 142, 507-538.

Gilbert Baumslag (1971), 'A finitely generated infinitely related group with trivial multiplicator', Bull. Austral. Math. Soc. 5, 131-136.

Gilbert Baumslag (1972), 'Some remarks about multiplicators and finitely presented groups', Math. Z. 126, 239-242.

Gilbert Baumslag (1974), 'Finitely presented metabelian groups', Proc. Second Internat. Conf. Theory of Groups, Canberra, 1973, 65-74 (Lecture Notes in Mathematics, 372, Springer-Verlag, Berlin, Heidelberg, New York).

Gilbert Baumslag and Ralph Strebel (in press), 'Some finitely generated, infinitely related metabelian groups with trivial multiplicator', J. Algebra.

A. K. Bousfield and D. Kan (1971), 'Homotopy Limits, Completions and Localizations', (Lecture Notes in Mathematics 249, Springer-Verlag, Berlin, Heidelberg, New York).

C. K. Gupta (1969), 'A faithful matrix representation for certain centre-by-metabelian groups', $J$. Austral. Math. Soc. 10, 451-464.

B. Н. Ремеслеунниов [V. N. Remeslennikov] (1969), 'Представленке конечно порожденных метабелеВыж групп матрнцамн- [Representaion of finitely generated metabelian groups by matrices], Algebra $i$ Logika 8, 72-75; Algebra and Logic 8, 39-40.

А. Л. Шмевькин [A. L. Šmel'kin] (1965), 'О разрешимых пронзведениях групп' [On soluble products of groups], Sibirsk. Mät. Ž. 6, 212-220.

City College of the City University of New York, Convent Avenue at 138th Street,

New York, U.S.A. 